\title{
Sexual health care training needs of general practitioner trainers: a regional survey
}

\author{
Wolfgang A Markham, Alison D Bullock, Philippa Matthews, Vickie R Firmstone, Stephen Kelly, Steve J Field
}

\section{Abstract}

Objectives The National Strategy for Sexual Health and HIV aims to facilitate improved patient access to sexual health care, primarily in general practice. This study aimed to identify sexual health care provision in general practitioner (GP) training practices and highlight training and resource implications of the strategy for GPs and prospective GPs.

Methods Data were gathered from interviews with five key representatives (all of whom had a special interest in GP training and/or sexual health care) and a self-completed questionnaire survey of all 374 GP trainers in the West Midlands region. The questionnaire was developed from the interviews and comprised three sections: sample characteristics; current practice; and 30 statements to elicit attitudes, knowledge and training implications. The questionnaire was mailed out in March 2002 with two remailings at 2-week intervals.

Results Most GP trainers $(79 \% ; n=295)$ returned completed questionnaires. Most respondents were already offering some 'Level 1 services' or were prepared to including cervical screening $(100 \% ; n=295)$, sexual history taking $(95 \% ; n=271)$, sexually transmitted infection (STI) testing (74\%; $n=217)$, HIV testing $(68 \% ; n=198)$ and contraceptive services $(71 \% ; n=208)$. However, most $(86 \% ; n=251)$ needed further information on the Strategy detail and its implications. Training needs in sexual history taking, STI testing and HIV testing were also highlighted. Most GP trainers $(62 \% ; n=181)$ believed GP registrars were relatively unprepared for sexual health care and proposed improved training and assessment. Appropriate nurse training should also be provided.

Conclusion Although $82 \%(n=242)$ of respondents would implement the Strategy if properly resourced, considerable training and support needs were identified.

J Fam Plann Reprod Health Care 2005; 31(3): 213-218 (Accepted 1 February 2005)

Key message points
- Most of the sample were already offering Level 1 services
or were prepared to do so in the future.
- Training needs were identified concerning the detail and
implication of the Strategy and also in the areas of sexual
history taking and STI and HIV testing.
- Nurses can play a key role in service provision and should
be given appropriate training. Over three-quarters of the
sample wanted to become fully involved in the National
Strategy providing it was resourced properly.

School of Health and Social Studies, University of Warwick, Coventry, UK

Wolfgang A Markham, BSc, PhD, Lecturer

School of Education, University of Birmingham, Birmingham, UK

Alison D Bullock, BA, PhD, Senior Research Fellow

Vickie R Firmstone, BA, PhD, Research Fellow

West Midlands Deanery, Birmingham, UK

Philippa Matthews, MBBS, FRCGP, Programme Director for HIV and Sexual Health

Stephen Kelly, FRCP, FRcGP, Director, Postgraduate General Practice Education

Steve J Field, MMEd, FRCGP, Postgraduate Dean

Correspondence to: Dr Wolfgang Markham, School of Health and Social Studies, University of Warwick, Coventry CV4 7AL, UK. E-mail: wolfgang.markham@warwick.ac.uk

\section{Introduction}

Context

A comprehensive National Strategy for Sexual Health and HIV was introduced in England in 2001. ${ }^{1}$ It focuses on the provision of sexual health care in primary care settings and more specialist provision and has three levels of service (Box 1). Level 1 elements are to be offered by general practitioners (GPs) and, where appropriate, nurses and should, it was proposed, be readily available in primary care settings to all patients. If general practices do not provide Level 1 service elements, it was proposed that they should be able to refer patients to other local primary care settings that do provide these services. Primary care teams with a special interest in sexual health are to be supported in providing Level 2 services. The main intention of the Strategy, therefore, is to facilitate improved patient access to sexual health care, primarily in primary care settings.

Training implications of the National Strategy for Sexual Health and HIV

The Strategy may have training implications, particularly as knowledge, skills and awareness of issues surrounding sexual health care vary amongst primary health care workers. ${ }^{2}$ There is evidence, for example, that some GPs may be insufficiently familiar with common tests for sexually transmitted infections (STIs) to be able to use them appropriately. $3-5$

A number of relevant training courses are available for qualified GPs. These courses include the Diploma of the

\begin{tabular}{|c|}
\hline $\begin{array}{l}\text { Box } 1 \text { Levels of service } \\
\text { Level } 1 \\
\text { - Sexual history and risk assessment } \\
\text { - STI testing for women } \\
\text { - HIV testing and counselling } \\
\text { - Pregnancy testing and referral } \\
\text { - Contraceptive information and services } \\
\text { - Assessment and referral of men with STI symptoms } \\
\text { - Cervical cytology screening and referral } \\
\text { - Hepatitis B immunisation } \\
\text { Level } 2 \\
\text { - Intrauterine device insertion } \\
\text { - Contraceptive implant insertion } \\
\text { - Testing and treating STIs } \\
\text { - Partner notification } \\
\text { - Vasectomy } \\
\text { - Invasive STI testing for men } \\
\text { Level } 3 \\
\text { - Outreach for STI prevention } \\
\text { - Outreach contraceptive services } \\
\text { - Specialised infections management, including co } \\
\text { - Highly specialised contraception } \\
\text { partner notification } \\
\text { - Spexialised HIV treatment and care } \\
\text { - }\end{array}$ \\
\hline
\end{tabular}


Box 2 Survey questions

- Testing for sexually transmitted infections, HIV testing, sexual history taking, contraceptive implant insertion ${ }^{\mathrm{a}}$ (Please tick one statement that is closest to what is done in your practice: We have done this, We have not done this but are prepared to in the future, We do not have the scope to do this in our practice, We do not think this is appropriate for general practice)

- It's easy to get high quality contraceptive care for my patients ${ }^{b}$ (Agree, Disagree, Don't know)

- In your practice, who takes cervical smears?c (GP, practice nurse, nurse practitioner)

- Screening for chlamydia prior to IUD insertion (Option: we do not fit IUDs)

- I have problems getting vasectomies for my patients ${ }^{\mathrm{e}}$ (Agree, Disagree, Don't know)

- Men who present with possible symptoms of STIs should be referred by the GP to a specialist GUM clinic

(Agree, Disagree, Don't know)

The superscript letters relate to the parameters in Table 3.

Faculty of Family Planning (DFFP) and the British Association of Sexual Health and HIV (BASHH, formerly the Medical Society for the Study of Venereal Disease) foundation course in STIs. Other courses, for example, the Sexual Health in Practice (SHIP) course in Birmingham, focus specifically on the learning needs of GPs and practice nurses. Additionally, the Royal College of Nursing has developed a distance-learning package for nurses related to the delivery of Level 1 services.

GP registrars may receive training in sexual health care via four main routes: during undergraduate training; in hospitals where senior house officers (SHOs) may undertake a placement in obstetrics and gynaecology; in family planning clinics; and in general practice. Having said this, overall, undergraduate training in sexual health care is variable and sometimes minimal. Placements in obstetrics and gynaecology do not usually include training in contraception and wider sexual health issues such as STIs. Family planning clinics may not focus on wider sexual health issues and tend not to teach the opportunistic approaches appropriate for primary care. ${ }^{6}$ Additionally, GP vocational trainees only occasionally have placements in genitourinary medicine and the GP registrar curriculum has many competing key aspects of practice. ${ }^{7}$ Wall and Houghton $^{6}$ raised concerns about the capacity of the MRCGP assessments to assess knowledge, abilities, skills and attitudes in contraception sufficiently, and this view could be extended to include wider sexual health care issues.

In summary, the sexual health care services that general practices are expected to offer as part of the National Strategy for Sexual Health and HIV did not clearly match the training programmes (and thus, qualifications) available when the study was undertaken.

\section{Study aims}

This study aimed to identify sexual health care practice amongst GP trainers in the West Midlands in 2002 and to highlight training and resource implications of the new sexual health care strategy. Sexual health care in this study included cervical screening, contraception, termination of pregnancy, STIs and HIV.

\section{Methods}

Data were gathered from interviews with five key representatives and a self-completed questionnaire survey of all GP trainers in the West Midlands. The five interviewees had a special interest in GP training and/or sexual health care. The interviewees included the Director of Postgraduate GP Education for the West Midlands Deanery, a senior clinical medical officer and DFFP course organiser, an advisor to Birmingham Health Authority on sexual health and HIV in primary care (and latterly the Programme Director for HIV and Sexual Health for the West Midlands Deanery) and two Area Directors of Postgraduate GP Education, one of whom is also a family planning instructing doctor. Two of the five interviewees were subsequently involved in the design of the questionnaire but did not take part in data analysis or initial drafting of this paper. As co-authors of this paper their contribution is recognised.

The questionnaire was developed from the interviews and comprised three sections: sample characteristics; current practice; and 30 statements to elicit attitudes, knowledge and training implications. It was first mailed out to all GP trainers $(n=374)$ in the West Midlands region (covering the Strategic Health Authority areas of Birmingham and Black Country, Coventry, Warwickshire, Herefordshire and Worcestershire, and Shropshire and Staffordshire) in March 2002 with two re-mailings at 2week intervals.

Data input errors were eliminated through basic frequencies and double-checking 20\% $(n=59)$ of the returns. The questionnaire data were analysed (basic frequencies and cross tabulations) using SPSS (Statistical Package for the Social Sciences, SPSS Inc., Chicago, IL, USA). The Chi-square test of statistical significance was used to examine relationships between key characteristics of the sample and responses to the questions and also interrelationships between responses. The hypotheses being explored were (1) are participant characteristics related to service provision and responses to the 30 statements and (2) are particular attitudes and answers to knowledge questions related to service provision and training needs. The 30 statements had six response categories (Strongly agree to Strongly disagree) and a separate 'Don't know' category. All three agreement categories were collapsed to one category, as were the three disagreement categories. We re-categorised the data in order to simplify the data and to enable us to legitimately use the Chi-square test of statistical significance.

\section{Results}

Survey response rate

Most $(79 \% ; n=295)$ of the total sample $(n=374)$ returned completed questionnaires.

\section{Sample characteristics}

Our sample comprised $72 \%(n=211)$ males while the target population (GP trainers in the West Midlands) comprised $73 \%$ males. Most practices were large, with just one single-handed practice. The main characteristics of our sample are summarised in Table 1. Most had a list size of 8000 or more patients $(60 \% ; n=177)$, between four and six partners (headcount) $(62 \% ; n=182)$ and between two and five part-time nurses $(89 \% ; n=210)$. All bar one practice had at least one part-time nurse. The average length of booked appointments was 10 minutes for $67 \%(n=198)$ of practices and 7.5 minutes for $26 \%(n=77)$.

The training practices of most respondents offered enhanced levels of care of diabetes, asthma and coronary heart disease (Table 1). About half $(51 \%)$ of the respondents offered enhanced levels of care of women's health or sexual health (excluding 13\% who offered enhanced care in both) (Table 1). Most respondents (84\%) also reported that they had at least a general interest in contraception and sexual health (Table 2). 
ARTICLE

Table 1 Characteristics of the sample and enhanced levels of care

\begin{tabular}{lrr}
\hline Sample characteristic & $n$ & $\%$ \\
\hline List size (patients) & & \\
$<4000$ & 22 & 8 \\
$4000-8000$ & 95 & 32 \\
$>8000$ & 177 & 60 \\
Headcount (partners) & 46 & 16 \\
$<4$ & 182 & 62 \\
$4-6$ & 65 & 22 \\
$>6$ & & \\
Length of booked appointments (minutes) & 6 & 2 \\
5 & 77 & 26 \\
7.5 & 198 & 67 \\
10 & 11 & 4 \\
$>10$ & 2 & 1 \\
Open & & \\
Areas of enhanced levels of care & 255 & 86 \\
Diabetes & 240 & 81 \\
Asthma & 222 & 75 \\
Coronary heart disease & 167 & 57 \\
Child health surveillance & 134 & 45 \\
Women's health & 79 & 27 \\
Obstetrics & 74 & 25 \\
Elderly care & 73 & 25 \\
Mental health & 68 & 23 \\
Rheumatology & 53 & 18 \\
Sexual health & 5 & 2 \\
None of these & 31 & 11 \\
Other & &
\end{tabular}

Table 2 Level of interest in contraception and sexual health and knowledge about the National Strategy for Sexual Health and HIV

\begin{tabular}{lrr}
\hline Parameter & $n$ & $\%$ \\
\hline Level of interest in contraception and sexual health & & \\
$\quad$ I have a special interest & 58 & 20 \\
I have a general interest & 190 & 64 \\
I have a slight interest & 27 & 9 \\
I have no particular interest & 20 & 7 \\
Knowledge about the Strategy & & \\
I have detailed knowledge of its content & 9 & 3 \\
I have some knowledge of its content & 112 & 38 \\
I know that it exists but I don't know about its content & 95 & 32 \\
I don't know about it & 78 & 27 \\
\hline
\end{tabular}

\section{Current practice}

Questions/statements in the questionnaire have, where possible, been matched to Level 1 and Level 2 services in the National Strategy for Sexual Health and HIV and the overview this provides of current practice is presented in Table 3 . As shown in Table 3, Level 1 services and some
Level 2 services pertaining to cervical screening, contraception, termination of pregnancy, sexual history taking, STI testing and HIV testing, were often already being delivered.

Cervical screening. The entire sample of GP trainers $(n=$ 295) provided cervical cytology screening (Table 3 ).

Contraception. Most respondents $(71 \% ; n=208)$ reported that their practices provided high-quality specialist contraceptive care and $76 \%(n=217)$ already provided contraceptive implants or were prepared to in future (Table $3)$. However, $17 \%(n=50)$ had insufficient scope to provide contraceptive implants and $7 \%(n=21)$ thought it inappropriate. Most of the GP trainers in this study $(87 \%$; $n=256$ ) also obtained appointments for vasectomies relatively easily (Table 3 ).

Termination of pregnancy. Nearly two-thirds (64\%; $n=186$ ) of respondents had ready access to termination of pregnancies. However, 30\% $(n=86)$ had limited access, $4 \%(n=11)$ had no National Health Service (NHS) access and $3 \%(n=8)$ opted out for ethical reasons.

Sexual history taking. Almost all respondents $(95 \%$; $n=275$ ) either already took sexual histories or were prepared to in future (Table 3). However, time was a constraint and almost half $(48 \% ; n=173)$ reported insufficient time to take sexual histories. No significant relationship was found between average length of booked appointments and views regarding insufficient time to take sexual histories.

STI testing. Most respondents $(74 \% ; n=217)$ reported they already tested for STIs (other than HIV) or were prepared to (Table 3$)$. Eight percent $(n=24)$ indicated their practice did not have scope to test for STIs (other than HIV) and 18\% ( $n$ $=51$ ) thought testing for STIs (other than HIV) is inappropriate for general practice. Having said this, almost all respondents $(91 \% ; n=256)$ already screen for chlamydia prior to intrauterine contraceptive device (IUD) insertion or were prepared to and most $(73 \% ; n=269)$ thought diagnosing chlamydia an integral part of sexual health care in general practice. More than one in five GP trainers $(22 \%$; $n=106$ ) reported they tended not to see people with STIs in their practice. In addition, almost all respondents $(95 \% ; n=$ 279 ) believed men with possible symptoms of STI should

Table 3 A partial overview of sexual health care in the practices of general practitioner trainers

\begin{tabular}{lll}
\hline Parameter & $\begin{array}{l}\text { Already provided or were } \\
\text { prepared to in the future } \\
{[n(\%)]}\end{array}$ & $\begin{array}{l}\text { Had insufficient scope or } \\
\text { thought it inappropriate for } \\
\text { general practice } \\
{[n(\%)]}\end{array}$ \\
& $\begin{array}{l}\text { Don't know } \\
{[n(\%)]}\end{array}$ \\
\hline Level 1 & $275(95)$ & $16(5)$ \\
Sexual history taking and risk assessment & See below \\
STI testing for women & See below & $94(32)$ \\
HIV testing and counselling & $198(68)$ & Not known \\
Pregnancy testing and referral & Not known & $76(26)$ disagree \\
Contraceptive information and services & See below \\
Assessment and referral of men with STI symptoms & $208(71)$ agree & 0 \\
Cervical cytology screening and referralc & See below & Not known \\
Hepatitis B immunisation & $295(100)$ & \\
Level 2 & Not known & $15(5)$ \\
Intrauterine device insertion & & $71(25)$ \\
Contraceptive implant insertion & & $76(26)$ \\
Testing and treating STIs & & Not known \\
Partner notification & $267(95)$ & $37(13)$ disagree \\
Vasectomye & $217(75)$ & $13(4)$ \\
Invasive STI testing for men ${ }^{\mathrm{a}}$ & $217(74)$ & Not known \\
\hline
\end{tabular}

The superscript letters refer to the survey questions detailed in Box 2. STI, sexually transmitted infection. 


\section{ARTICLE}

be referred to departments of genitourinary medicine (GUM clinics) (Table 3). We may wonder whether women's sexual health care is favoured in general practice.

HIV testing. Perhaps more respondents than expected $(58 \% ; n=170)$ had already tested for HIV and a further $10 \%(n=28)$ were prepared to (Table 3$)$. Similar proportions of respondents believed it is inappropriate in general practice to test for HIV $(22 \% ; n=63)$ and STIs other than HIV $(18 \% ; n=51)$.

\section{Knowledge}

Some questions aimed to elicit knowledge levels. Most respondents $(65 \% ; n=190)$ knew that young women who have a termination of pregnancy are more likely than their age-matched peers to have received emergency contraception in the year before conception. ${ }^{8}$ However, quite a sizable proportion $(20 \% ; n=57)$ indicated that they did not know. Furthermore, only 12\% $(n=43)$ were aware that partner notification is not necessary for those patients who are diagnosed as having genital herpes and/or genital warts. Unfortunately, there are no effective treatments for genital herpes and genital warts that protect the patient from re-infection and inhibit the further spread of the infection into the community.

\section{General views on the National Strategy for Sexual Health and HIV}

Few respondents $(3 \% ; n=9)$ felt they had detailed knowledge of the National Strategy for Sexual Health and HIV at the time of the survey (Table 2). Most GP trainers $(59 \% ; n=173)$ were either unaware that the Strategy existed or were aware that it existed but did not know the content. Given GP workloads ${ }^{2}$ and the newness of the Strategy at the time of the survey, these findings should be viewed less as a criticism of GP trainers and more as an indication of training need. These findings may also indicate that at the time of our survey, there may have been a need for greater promotion of the National Strategy for Sexual Health and HIV by the Department of Health .

Most respondents $(73 \% ; n=213)$ believed the National Strategy to be yet another government initiative to be foisted on GPs who are commonly overstretched. Additionally, the majority of respondents $(67 \% ; n=194)$ believed most GPs would pay little attention to the new Strategy. Despite these findings, most respondents $(82 \% ; n=242)$ reported that they wanted their training practice to become fully involved with the National Strategy for Sexual Health and HIV provided that the Strategy was resourced properly.

\section{Training implications}

Given the relative newness of the Strategy at the time of the survey, it is unsurprising that most GP trainers (86\%; $n=251$ ) believed they needed training on its implications. Interestingly, statistically significantly more women wanted this training $(p \leq 0.01)$. However, a tendency not to see patients with STIs did not significantly influence a perceived need for training on the Strategy implications.

Training in specific areas of sexual health care was also requested. A sizeable proportion (44\% of those answering the question; $n=67$ ) wanted further training in STI (other than HIV) testing and 39\% (of those answering the question; $n=55$ ) wanted further training in HIV testing. For both STI (other than HIV) testing and HIV testing, statistically significantly more women wanted further training ( $p \leq 0.05$ and $p \leq 0.001$, respectively). There were no significant relationships between wanting training in either STI (other than HIV) testing or HIV testing and reporting a tendency not to see patients with STIs.
Most GP trainers $(69 \% ; n=202)$ believed that the initial IUD training and re-certification requirements discourage GPs from IUD insertion training.

More than half of the respondents $58 \%$ of those answering the question; $n=97$ ) reported that they wanted further training in sexual history taking. However, most $(75 \% ; n=220)$ were happy to teach their registrars' sexual history taking. More women $(p \leq 0.001)$ and respondents with a general/special interest in sexual health $(p \leq 0.01)$ were willing to teach their registrars' sexual history taking. Most respondents $(62 \% ; n=181)$ believed GP registrars were ill-prepared for sexual health care in general practice no matter what hospital jobs they had held. Most GP trainers $(75 \% ; n=225)$ also believed that an assessment of competence in contraceptive care should be mandatory in the MRCGP assessments.

\section{Discussion}

\section{Summary}

This study found a readiness amongst GP trainers in the West Midlands to act upon the National Strategy for Sexual Health and HIV. Further information on the strategy detail and its implications was needed. Additionally, specific training needs were identified regarding sexual history taking/risk assessment, HIV testing (including issues of confidentiality) and testing for STIs other than HIV. Most respondents were already offering Level 1 services or were prepared to. However, some respondents believed it inappropriate to test for HIV $(22 \% ; n=63)$ or STIs other than HIV $(18 \% ; n=51)$ in general practice. Some respondents also appeared selective about the STIs they thought appropriate to test and treat and most respondents appeared to favour women's sexual health care over men's sexual health care. Most GP trainers felt the system of IUD training and re-accreditation acted as a disincentive to learning this skill. Most thought GP registrars would be relatively unprepared for contraceptive and sexual health care and consideration should consequently be given to improving GP registrar training and assessment. Additionally, there was a broader concern that few GPs would heed the new National Strategy.

\section{Study strengths and limitations}

This study had a high response rate $(79 \% ; n=211)$. However, the views expressed may not represent the views of GPs who are not GP trainers. Additionally, the survey was confined to one region (the West Midlands) and the findings may not be representative of other regions.

\section{Sexual history taking, testing for STIs other than HIV and HIV testing}

Patients commonly feel uncomfortable, embarrassed, ashamed or humiliated when they talk about their sexual health and sexual problems. ${ }^{9-12}$ Additionally, patients in primary care settings may not expect to discuss sexual matters and their sexual experience. ${ }^{9}$ A sizeable proportion (58\% of those answering the question; $n=97$ ) of GP trainers (who responded to the question; $n=97$ ) wanted sexual history taking training which might suggest they were aware of the difficulties of obtaining appropriate sexual histories within primary care settings. However, further research is required to confirm this interpretation. In addition to wanting sexual history taking training, most respondents $(75 \% ; n=220)$ were also happy to teach their registrars sexual history taking. The positive interpretation of this finding is that most GP trainers have a willing approach to teaching their registrars. A concern is that some might have training needs themselves. Most respondents $(62 \% ; n=181)$ believed GP registrars were ill-prepared for 
sexual health care in general practice no matter what hospital jobs they had held. This finding, together with the observation that some GP trainers $(25 \% ; n=74)$ were not comfortable teaching their registrars sexual history taking, highlights potential training needs of GP registrars.

More than one in five GP trainers $(22 \% ; n=106)$ reported they tended not to see people with STIs in their practice. This may occur for three reasons. First, practices may have low STI prevalence. Some practices were in relatively affluent areas and less likely to have patients with STIs because inequalities in sexual health mirror inequalities in health. ${ }^{1}$ Second, some patients with STIs may choose to go a GUM clinic rather than their GP. Third, it is also possible that some practitioners may not recognise the more subtle symptoms of infection (such as slight intermenstrual bleeding) or they may not practice in a way that supports detection of asymptomatic infection.

The results regarding chlamydia screening and STI testing (other than HIV) are interesting because they highlight a potential degree of inconsistency. Hence, even though the vast majority of respondents $(91 \% ; n=256)$ already screen for chlamydia prior to IUD insertion, a proportion $(18 \% ; n=51)$ also reported that they thought testing for STIs is inappropriate in general practice. Almost half of the $18 \%$ who thought testing for STIs is inappropriate for general practice already screen for chlamydia prior to IUD insertion. Thus, approaching one in ten GP trainers either screen for chlamydia but believe it is inappropriate to do so in general practice and/or make a distinction between screening for chlamydia prior to IUD insertion and testing generally for STIs.

The National Strategy for Sexual Health and HIV aims to facilitate improved access to sexual health care of all patients. It may be that women's sexual health care appears to be favoured amongst the GP trainers in this study because general practices have a long-established role in cervical screening and the provision of contraception for women. Invasive STI testing for men is a Level 2 element. However, the observation that almost all respondents (95\%; $n=279$ ) believed men with possible symptoms of STI should be referred to GUM clinics is worth highlighting. This observation is even more interesting given that almost half of the responders $(45 \% ; n=133)$ were aware of attendance problems at GUM clinics following GP/practice nurse referral. Some studies report high GUM clinic attendance following referral ${ }^{13}$ whereas others observed low attendance rates. $9,14,15$ Accessing men generally in primary care contexts is problematic. ${ }^{16}$ In relation to women, men commonly perceive they are at less risk, ${ }^{17}$ are more likely to normalise symptoms, ${ }^{18}$ are more likely to fear wasting doctors' time ${ }^{19}$ and are more likely to experience structural and institutional barriers to health services in general. ${ }^{19}$ Thus, an almost unanimous preference for referring male patients to other agencies when they are already relatively difficult to access in general practice could be regarded as a lost opportunity and rather disappointing. Consideration of how the sexual health care of men may be promoted within general practice may be warranted. This view is consistent with the new General Medical Services (GMS) Contract (GMS2) ${ }^{20}$ which draws specific attention to effective communication with young men under enhanced sexual health care services.

We do not know why practices do or do not test for HIV. However, three attitudes may contribute to the apparent reluctance of some respondents to support HIV testing in general practice. First, some GP trainers $(40 \%$; $n=116$ ) believed GPs avoid HIV testing because providing support and care is stressful. GP trainers who believe HIV testing is inappropriate or do not have the scope were significantly more likely $(p \leq 0.01)$ also to believe that GPs avoid HIV testing because providing support and care is stressful. Second, most $(73 \% ; n=212)$ of the respondents believed telling a patient that she/he is HIV positive was worse than telling a patient she/he had multiple sclerosis. This finding is interesting given that the treatment of HIV is highly effective in comparison with that of multiple sclerosis. Third, most $(76 \% ; n=222)$ believed HIV, confidentiality and life assurance/insurance remains an issue. However, those who thought HIV testing in general practice was either inappropriate or did not have the scope were significantly less likely $(p \leq 0.05)$ to believe HIV, confidentiality and life assurance/insurance is an issue.

Confidentiality is a major issue for sexual health care and influences access to care. Confidentiality issues are, for example, a key concern for under-16s seeking contraceptive care in general practice. ${ }^{21}$ Most of the respondents $(76 \% ; n=222)$ believed confidentiality and life insurance/assurance remains a barrier to HIV testing. This view is supported by the Royal College of General Practitioners (RCGP). ${ }^{2}$ The UK is the only EU country that allows insurance companies to access comprehensive medical information from personal medical attendants. The $\mathrm{RCGP}^{2}$ believe the resolution of the HIV/insurance quandary would enable patients to feel confident of confidentiality in both GUM clinics and general practice. This would facilitate more widespread HIV testing, which is a key aim of the national sexual health care strategy.

\section{Implications for future clinical practice}

Three interviewees highlighted the role of nurses. One interviewee maintained Level 1 provision is straightforward, providing a general practice has a practice nurse and protocols in place. Nurses may provide contraceptive advice, offer free condoms (where practices are able to offer these), be the first point of contact for women with symptoms of STI, and take sexual histories. They may also discuss relationship difficulties and other aspects of sexual health promotion. The major barriers to nurse-led provision of sexual health care are time and lack of appropriate training 22 that is sufficiently resourced. Recognition should also be given to the problems of singlehanded practices, which do not enjoy the economies of scale that might enable them to take on nursing staff.

Most respondents $(67 \% ; n=194)$ thought few GPs would heed the National Strategy for Sexual Health and HIV. This finding is likely to be related to three factors. First, low morale in the medical and nursing professions. ${ }^{2}$ Second, our observation that nearly three-quarters of our study participants $(73 \% ; n=213)$ believed the new Strategy is yet another government initiative to be foisted upon GPs who are commonly overstretched. Third, the resources needed to implement the Strategy.

A recent House of Commons Health Committee report $^{23}$ also recorded that the successful implementation of the National Sexual Health and HIV Strategy requires adequate resources and considerable training and support. Although some doubted that sufficient resources would be found, it was hoped that resource implications might be addressed in the new GMS2.20 The GMS2 categorises contraception and cervical screening as additional services and GP practices will normally be expected to provide these additional services. However, other aspects of sexual health, including some Level 1 elements such as STI testing, are categorised as national enhanced services. Many feel that there is a lack of congruence between the National Strategy for Sexual Health and HIV and the GMS2 because some Level 1 elements such as STI testing 


\section{ARTICLE}

are categorised as national enhanced services. This view contrasts with the view of the Department of Health Sexual Health Team who maintain that GMS2 does not conflict with the National Strategy for Sexual Health and HIV and the views of Gafos. ${ }^{24}$ Gafos, in a recent editorial, put forward the case that 'essential services' equate with Level 1 elements of the National Sexual Health and HIV Strategy, and that enhanced services equate to Level 2 elements of the National Strategy. ${ }^{24}$ There are other concerns regarding the new GMS2. Some argue that the separation of contraception and cervical screening from enhanced sexual health care services in the GMS2 does not facilitate an integrated approach to sexual health care. So, for example the GMS2 considers monitoring contraceptive care follow-up to termination of pregnancy to be an enhanced service but no other aspect of contraception is considered an enhanced service.

The new GMS2 does include funding for national enhanced services such as 'IUD fitting' and 'more specialised sexual health services'. This means funding will be available for the fitting, monitoring, checking and removal of IUDs and related chlamydia screening, condoms and pregnancy testing kits, HIV testing, including pre/post-test counselling, STI screening and treatment, and staff training. Having said this, accessing funding for sexual health care that is categorised as an enhanced service is dependent on approval by local primary care organisations (PCOs). Local PCOs do consult constituent local general practices, local medical committees and patient forums regarding the local priority of sexual health care and the level of financial support for enhanced sexual health care services. Nevertheless, few PCOs are currently commissioning enhanced sexual health care services. This is disappointing given that most respondents in this study $(82 \% ; n=242)$ reported that they wanted their training practice to become fully involved in the national sexua health care strategy providing that it was adequately resourced. It is possible that some GP trainers in this study who were offering and continue to offer some Level sexual health care services may not be currently resourced for these services by their local PCO. It is also possible that other respondents may have ceased to offer some Level 1 services because of lack of resources. It is hoped that this is not the case, as a primary aim of the new GMS2 is to facilitate increased autonomy amongst GPs, and thus greater choice regarding the services they provide. The categorisation of some Level 1 elements, such as STI testing, as enhanced services, also raises the possibility that some Level 1 elements will not be readily available in primary care settings for some patients in some localities because of lack of resources. This appears contrary to the aims of the national sexual health care strategy.

Many Level I services that are categorised as national enhanced services in the GMS2 such as STI testing are new to general practice and will not be implemented without education and resources. In Birmingham, PCOs are trying to achieve this by commissioning the SHIP scheme, which provides training and resources for practices. PCOs that do decide to commission enhanced sexual health care services face two additional levels of difficulty. First, assessing the quality of sexual health care delivered by primary care clinicians may not be straightforward. Thus, for example how will being "able to communicate effectively with all young people including young men, gay and lesbian people and ethnic minorities" 20 be assessed? Second, PCOs need to clarify what constitutes evidence of an acceptable level of educational attainment that is relevant to primary care. It is hoped that those PCOs who intend to commission enhanced sexual health care services in the future may benefit from the lessons learned from the relatively few PCOs that are currently commissioning these services.

Some have suggested that the National Strategy for Sexual Health and HIV never achieved the status it deserved. Even so, until sexual health reaches National Service Framework status, there will inevitably be ongoing geographical variability in sexual health care services.

\section{Acknowledgements}

The project team would like to thank all the study participants. They are grateful for the financial support of the NHS West Midlands. The views and opinions expressed are the authors' own and do not necessarily reflect those of the funders. Special thanks also go to Dr David Wall for his critical comments on the draft project report.

Statements on funding and competing interests

Funding. This study was funded by West Midlands NHS

Competing interests. None identified.

References

1 Department of Health. The National Strategy for Sexual Health and $H I V$. London, UK: Department of Health, July 2001.

2 Royal College of General Practitioners (RCGP). Response to the National Sexual Health and HIV Strategy. London, UK: RCGP, January 2002

3 Ainsworth JG, Weaver T, Murphy S, Renton A. General practitioners mmediate management of men presenting with urethral problems. Genitourin Med 1996; 72: 427-430.

4 Nunns D, Mandal D. The chronically symptomatic vulva: prevalence in primary health care. Genitourin Med 1996; 72: 343-344.

5 Stokes T, Bhaduri S, Schober P, Shukla R. GPs' management of genital chlamydia: a survey of reported practice. Fam Pract 1997; 14: 455-460.

6 Wall D, Houghton G. Family planning - what needs to be taught? A view from general practice in the West Midlands, UK $B r J$ Fam view from general practic

7 MRCGP Clinical Medicine Curriculum for GP Registrars (The Wolverhampton Grid). http://www.wmdeanery.org/downloads/pdf/ wtongrid1999.pdf [Accessed 6 June 2005].

8 Churchill D, Allen J, Pringle M, Hippisley-Cox J, Ebdon D, Macpherson $\mathrm{M}$, et al. Consultation patterns and provision of contraception in general practice before teenage pregnancy: case control study. BMJ. 2000; 321: 486-489.

9 Matthews P, Fletcher J. Sexually transmitted infections in primary care: a need for education. Br J Gen Pract 2001; 51: 52-56.

10 Nicholson S, Carter YH, Savage W, Collinson S. Let's talk about sex: a description of an interactive sexual history teaching session. Med Teach 2000, 22: 412-414.

11 Baraitser P, Elliot L, Bigrigg A. How to talk about sex and do it well: a course for medical students. Med Teach 1998; 20: 237-240

12 Matthews P. Sexual history taking in primary care. In: Carter Y, Moss C, Weyman A (eds), RCGP Handbook of Sexual Health in Primary Care. London, UK: RCGP, 1998; Chapter 2, 17-50.

13 Rogstad KE, Kellock DJ, Horton M, Kudesia G. Genital chlamydia trachomatis infections in primary care. Genitourin Med 1997; 23: 224

14 Tobin JM, Bateman J, Banks B, Jeffs J. Clinical audit of the process of referral to genitourinary medicine of patients found to be chlamydia positive in a family planning service. Br J Fam Plann 1999; 24: 160-163.

15 Wilkinson C, Massil H. An interface of chlamydia testing by community family planning clinics and referral to hospital genitourinary medicine clinics. Br J Fam Plann 2000; 26: 206-209.

16 Jewel D. Primary care. In: Davidson N, Lloyd T (eds), Promoting Men's Health: A Guide for Practitioners. London, UK: Bailliere Tindall, 2001

17 Blaxter M. Health and Lifestyles. London, UK: Routledge, 1990.

18 Bendelow G. Pain perceptions, emotions and gender. Sociol Health Illn 1993; 15: 273-294.

19 Men's Health Forum. Getting it Sorted: A Policy Programme for Men's Health. London, UK: Men's Health Forum, 2004

20 National Health Service Confederation and British Medical Association (BMA). New GMS Contract 2003. London, UK: BMA. 2003.

21 British Medical Association. Confidentiality and People Under 16 Guidance issued jointly by the BMA, GMSC, HEA, Brook Advisory Centres, FPA and RCPG, January 1994.

22 Stokes T, Mears J. Sexual health and the practice nurse: a survey of reported practice and attitudes. Br J Fam Plann 2000; 26: 89-92.

23 House of Commons Health Committee. Sexual Health: Third Report of Session 2002-03. London, UK: The Stationery Office, 2003.

24 Gafos M. The new General Medical Services (GMS) contract and the National Strategy for Sexual Health and HIV in England. $J$ Fam Plann Reprod Health Care 2004; 30: 147-149. 